\title{
Universiteit
}

Leiden

The Netherlands

\section{MEMS-based high speed scanning probe microscopy}

Disseldorp, E.C.M.; Tabak, F.C.; Wortel, G.H.; Katan, A.J.; Hesselberth, M.B.S.; Oosterkamp, T.H.; ... ; Spengen, W.M. van

\section{Citation}

Disseldorp, E. C. M., Tabak, F. C., Wortel, G. H., Katan, A. J., Hesselberth, M. B. S., Oosterkamp, T. H., ... Spengen, W. M. van. (2010). MEMS-based high speed scanning probe microscopy. Review Of Scientific Instruments, 81(4), 043702. doi:10.1063/1.3361215

Version: $\quad$ Not Applicable (or Unknown)

License: $\quad$ Leiden University Non-exclusive license

Downloaded from: https://hdl.handle.net/1887/61345

Note: To cite this publication please use the final published version (if applicable). 


\title{
MEMS-based high speed scanning probe microscopy
}

\author{
E. C. M. Disseldorp, F. C. Tabak, ${ }^{a)}$ A. J. Katan, ${ }^{\text {b) }}$ M. B. S. Hesselberth, T. H. Oosterkamp, \\ J. W. M. Frenken, and W. M. van Spengen ${ }^{\text {c) }}$ \\ Leiden University, Niels Bohrweg 2, 2333 CA Leiden, The Netherlands
}

(Received 18 December 2009; accepted 22 February 2010; published online 12 April 2010)

The high speed performance of a scanning probe microscope (SPM) is improved if a microelectromechanical systems (MEMS) device is employed for the out-of-plane scanning motion. We have carried out experiments with MEMS high-speed z-scanners (189 kHz fundamental resonance frequency) in both atomic force microscope and scanning tunneling microscope modes. The experiments show that with the current MEMS z-scanner, lateral tip speeds of $5 \mathrm{~mm} / \mathrm{s}$ can be achieved with full feedback on surfaces with significant roughness. The improvement in scan speed, obtained with MEMS scanners, increases the possibilities for SPM observations of dynamic processes. Even higher speed MEMS scanners with fundamental resonance frequencies in excess of a megahertz are currently under development. () 2010 American Institute of Physics.

[doi:10.1063/1.3361215]

\section{INTRODUCTION}

\section{A. High speed scanning probe microscope with piezoscanners}

High-speed, high-resolution imaging is of fundamental importance for studying dynamic processes, e.g., dynamic biomolecular processes or catalytic reactions in industrial applications. The advantages of scanning probe microscope (SPM) imaging with respect to other nanoscale imaging techniques are its high resolution, the applicability under many different circumstances (under vacuum, in liquid, etc.), and the nondestructive nature of scanning with feedback. Nevertheless, the limited scan speed is problematic since reaction rates in both biophysical and catalytic reactions are high. The reaction rates can be lowered by cooling the system to cryogenic temperatures, but this may change the reaction mechanism or completely stop the reaction. Therefore, fast imaging is required to get a better understanding of fast processes such as those mentioned above.

The state-of-the-art high speed atomic force microscope (AFM) imaging using piezos with feedback has been reported by Yamashita et al., ${ }^{1}$ Fukuma et al., ${ }^{2}$ and Schitter et $\mathrm{al}^{3}$ Maximum scan speeds are in the $\mathrm{mm} / \mathrm{s}$ and tens of frames/s range. Picco et al. ${ }^{4}$ showed that for AFM scanning without feedback the frame rate can be increased to 1300 frames/s. Powerful as this may be, the invasive nature of scanning without feedback makes that this technique cannot be used in all applications. High speed scanning tunneling microscopy (STM) imaging has been shown by Rost et al. ${ }^{5}$ up to a tip speed of $0.3 \mathrm{~mm} / \mathrm{s}$ on a clean $\mathrm{Cu}(001)$ surface with a scan size of $240 \times 240 \mathrm{~nm}^{2}$ and $512 \times 512$ pixels.

Although high-speed scanning with feedback using pi-

\footnotetext{
${ }^{\text {a) }}$ Author to whom correspondence should be addressed. Electronic mail: tabak@physics.leidenuniv.nl.

${ }^{b)}$ Present address: Lawrence Berkeley National Laboratory, 1 Cyclotron Road, Berkeley, California, USA.

${ }^{c}$ Also with Falco Systems, Gelderlandplein 75L, 1082 LV Amsterdam, The Netherlands
}

ezos is already very powerful, piezobased microscopes are still limited by the fundamental resonance frequency and the mass of the scanning piezoelement. At high scanning speeds the tip-sample distance cannot be changed sufficiently quickly to follow the surface topology due to the bandwidth limit of the piezoscanner. In addition, the piezoelement represents a significant mass that moves quickly in the whole microscope assembly, which tends to excite resonances in other parts of the mechanical loop from tip to sample. This typically results in image deformation at frequencies well below the actual piezoresonance frequency.

We have developed a high speed microelectromechanical systems (MEMS) scanner that circumvents these limitations of piezoscanners. MEMS have the advantage that their resonance frequency can be higher while having the same displacement range. Also, because MEMS are microscopic devices, their mass is negligible compared with the masses present in the rest of the microscope. Hence, the moving MEMS scanner will not excite any resonances in the mechanical loop. Of the three scanning directions, the out-ofplane z-direction requires the highest resonance frequency because it has to follow the full surface topology (roughness, rattling over individual atoms, and so on), while in the $\mathrm{x}$ - and $y$-directions the scanner merely has to be able to scan a square line by line. For that reason it is most advantageous to focus with the MEMS scanner on the motion in the z-direction, while a conventional piezo can take care of the slower $\mathrm{x}$ - and $\mathrm{y}$-directions. The first generation of MEMS $z$-scanner devices already offers performance on par with the fastest piezoscanners available.

The requirement of a fast $z$-scan as compared with $x$ and $y$ can be illustrated as follows. A protein molecule has to be imaged undergoing some structural change on the $10 \mu \mathrm{s}$ time scale and the height change in the $z$-direction is $2 \mathrm{~nm}$. To prevent the tip from crashing into this changing molecule, we want to move the tip upward to $2 \mathrm{~nm}$ within $10 \mu \mathrm{s}$. This means that the scanner has to handle an acceleration of $\mathrm{a}=2 \times 2 \times 10^{-9} /\left(10 \times 10^{-6}\right)^{2}=40 \mathrm{~m} /\left(\mathrm{s}^{2}\right)=4 \mathrm{~g}$. We could 
say that for the tip not to crash, the feedback system (including the tip) has to react within the first $1 \mu$ s. This corresponds to a bandwidth of $1 \mathrm{MHz}$. The $x$ and $y$ can continue unaltered, but the $z$-direction has to respond quickly to the changing condition.

One should take care, however, that a sufficient $z$-speed, while required for obtaining high-resolution images, is not a sufficient condition. The shape of the tip also influences the image quality because the image is a convolution of the sample being imaged and the tip shape. Hence, sufficiently sharp and durable tips should be used in addition to a fast scanner.

\section{B. MEMS SPM scanners}

The new MEMS SPM scanners have high resonance frequencies in the $200 \mathrm{kHz}$ range and an extremely low mass (order of magnitude $10^{-11} \mathrm{~kg}$, a factor of $10^{8}$ less than a scanning piezoelement). They can thus be used safely in any SPM setup; the MEMS scanner will not excite any resonances in the mechanical loop from tip to sample due to the large ratio between the fixed and moving masses.

Previous work on MEMS SPM has not been focused on high-speed imaging with feedback, but mainly on parallel scanning, as in the IBM millipede project, ${ }^{6}$ and on the integration of full three-dimensional scanning. A MEMS SPM device has been presented by Indermuehle et $a{ }^{7}{ }^{7}$ as early as 1994, and in 1995, the first AFM images were published, made with a slightly adjusted design of an x,y-microactuator with integrated tip..$^{8} \mathrm{Xu}$ et al. ${ }^{9}$ presented a working MEMS STM x,y,z-scanner as well. Apart from the millipede device, which functions very different from a normal AFM or STM, none of these scanners was optimized for high speed performance.

Our MEMS z-scanners have a scan range of several hundreds of nanometers in the vertical direction and can be actuated electrostatically at several hundred kilohertz to over a megahertz, depending on the exact design. MEMS z-scanners can be combined with a conventional piezo-zscanner (to extend its vertical range) or can replace it altogether when studying smooth surfaces.

\section{THE HIGH SPEED MEMS Z-SCANNER}

\section{A. The MEMS scanner geometry}

MEMS devices are micrometer-sized moving structures that are made using production techniques which were adapted from those used in the microelectronics industry. The polycrystalline silicon (polysilicon) MEMS z-scanner die has been produced in the PolyMUMPs process ${ }^{10}$ and consists of a movable membrane held by four springs. ${ }^{11}$ The springs are anchored to the substrate via short pillars, which suspend the membrane at $\sim 2 \mu \mathrm{m}$ above the actuation electrode. In Fig. 1, we show a scanning electron microscope (SEM) micrograph of a typical device [Fig. 1(a)] as well as a cross section showing the electrostatic actuator under the movable membrane more clearly [Fig. 1(b)].

A voltage difference applied between the membrane and actuator will exert an attractive electrostatic force between the two and pull the membrane toward the actuator. Variation

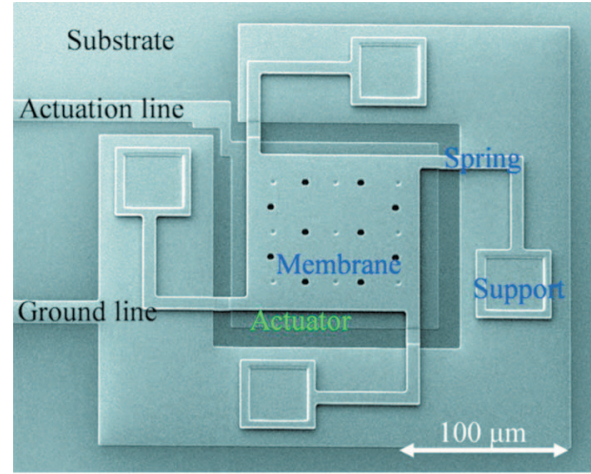

(a)

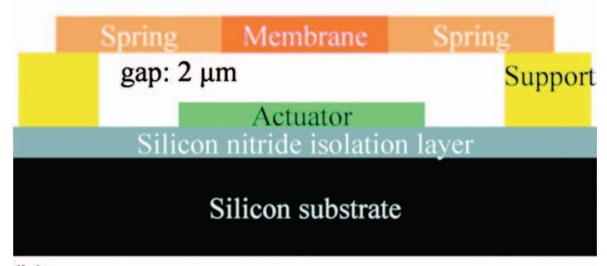

(b)

FIG. 1. (Color) (a) SEM micrograph of a MEMS z-scanner. (b) Simplified side view of MEMS z-scanner. The supports and springs ( $2 \mu \mathrm{m}$ thick) suspend the membrane $2 \mu \mathrm{m}$ above the actuator plate. The membrane is typically $2 \mu \mathrm{m}$ thick and tens of microns wide. The polysilicon structure of the z-scanner is deposited on a silicon nitride substrate, which electrically isolates the different parts of the MEMS.

in the applied actuation voltage results in a controlled displacement of the membrane. This displacement is used to control the tip-sample distance during scanning. The MEMS $\mathrm{z}$-scanner is mounted on a conventional $\mathrm{x}, \mathrm{y}$-piezoscanner which provides the lateral scan motion.

\section{B. Vertical scan range}

The required actuation voltages and the maximum scan range can be estimated by calculating the force on the scanner membrane. We model the membrane and the corresponding actuation plate as a parallel plate capacitor. Although the actual force is $\sim 20 \%$ higher due to fringe fields, this approximation already gives a good indication of the behavior of the scanner. The force $F$ in the parallel plate approximation is given by ${ }^{12}$

$$
F=-\frac{\varepsilon_{0} A}{(g-d)^{2}} V_{\mathrm{act}}^{2},
$$

where $V_{\text {act }}$ is the actuation voltage, $g$ is the gap between the actuator and membrane at zero voltage difference, $d$ is the displacement of the membrane from the initial position, $A$ is the surface overlap of the membrane and the actuator, and $\varepsilon_{0}$ is the permittivity of free space.

The force $F$ results in a displacement $d$ of the membrane dictated by the actuation voltage force [Eq. (1)] and the spring force exerted on the membrane by the supporting legs,

$$
F=k d \text {. }
$$

For small $V_{\text {act }}$, the membrane displacement $d$ of the scanner scales roughly quadratically with $V_{\text {act }}$, but as the separation between the membrane and actuator becomes smaller, the $d$ in the denominator makes the force grow quicker than qua- 
dratic. After the membrane has traveled one third of the initial gap $g$, this causes a "pull-in" event. This is a catastrophic event due to the following reason. Due to the high voltage between the actuator and the moving membrane, there is a significant charge accumulated on the two plates of the capacitor formed by the MEMS scanner. When the membrane collapses, it lands directly on top of the actuator. The charge is then short circuited and causes a high current spike that welds the membrane to the actuator. Even when the plates would not completely weld, the reliability of the MEMS scanner may be compromised upon pull-in: surface forces like capillary condensation and molecular van der Waals forces can, on this scale, be powerful enough to keep the membrane in a permanently stuck condition. The pull-in at $1 / 3$ of the gap size is hence the limiting factor of the vertical range of the MEMS z-scanner.

Charging of the dielectric silicon nitride layer used in the PolyMUMPs process to electrically isolate the different parts of the device from the wafer substrate is a commonly known problem in MEMS at high actuation voltages. It depends on the properties of the dielectric layer and the electric field strength. ${ }^{13}$ When charges accumulate in the dielectric, the charge on the actuator is no longer the only factor determining the position on the membrane: the accumulated charge in the silicon nitride layer will exert an extra force on the scanning membrane and hamper the proper motion of the scanner. We have experimentally verified that charging does not influence the operation of the MEMS z-scanner with actuation voltages up to $80 \mathrm{~V}$. This sets a second constraint for the vertical scan range. Either the scan range is limited due to pull-in at $1 / 3$ of the total gap between the plates, or, if the scanner is very stiff, the scan range is limited by the maximum actuation voltage instead.

\section{Simulation and experimental verification of the MEMS scanner motion}

In Fig. 2(a), the results of finite element model (FEM) simulations and experimental data are given for the displacement $d$ of the membrane as a function of $V_{\text {act }}$ using a series of MEMS z-scanners with different geometries. The experimental values were obtained by monitoring the height change in the membrane upon actuation in a conventional AFM, while the FEM simulations were done with the COMSOL structural mechanics package. In the model, a force per area, given by dividing $F$ [Eq. (1)] by the surface overlap $A$, acts on the membrane. The results of this calculation are within $15 \%$ of the experimental values. The model is therefore sufficiently accurate to be used to predict the actuation response curve and the pull-in voltage of actual MEMS devices.

From Fig. 2(a) we also see that the scanners used in the experiments have a pull-in voltage of $\sim 40 \mathrm{~V}$, and therefore their motion will not be distorted by charging effects.

The resonance frequencies of the MEMS $\mathrm{z}$-scanners [Fig. 2(b)] were also calculated with COMSOL. For the devices $\mathrm{A}$ and $\mathrm{B}$, a value of $218 \mathrm{kHz}$ was found, which is comparable to the very best piezostacks currently available for the purpose. Experimental verification with an optical technique ${ }^{14}$ shows a very clear, smooth response with a fun-
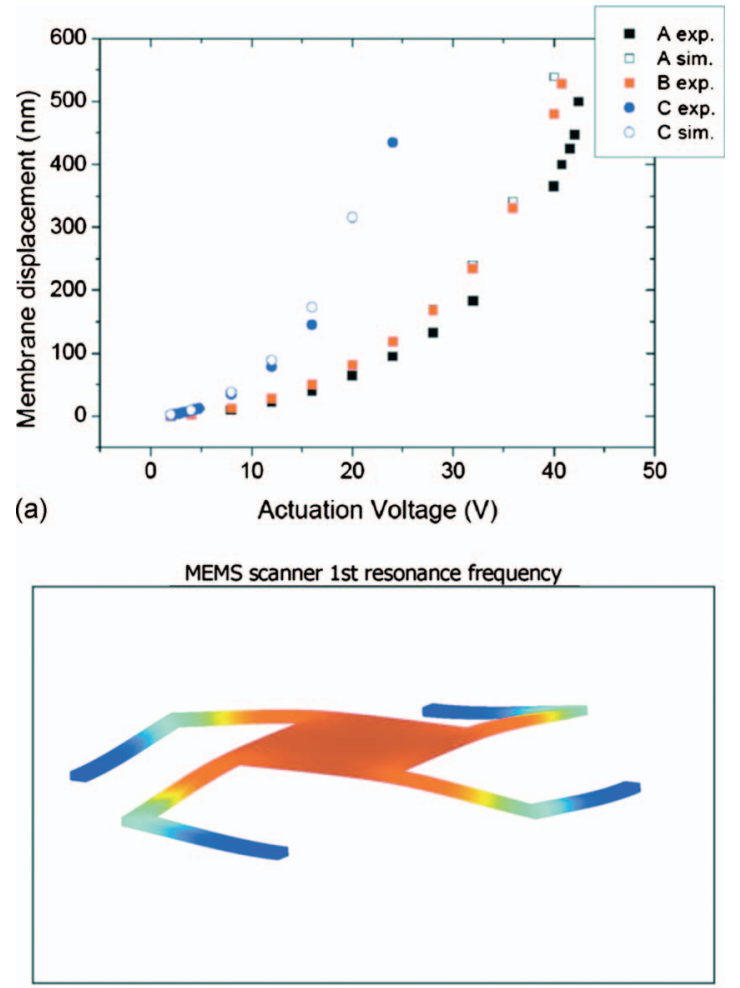

(b)

FIG. 2. (Color) (a) Membrane displacement $d$ (nanometer) as a function of actuation voltage $V_{\text {act }}$ (volt). Shown are both FEM simulations (indicated as "sim") and experimental values (indicated as "exp"). Devices A and B have equal dimensions $\left(60 \times 60 \mu \mathrm{m}^{2}\right.$ membrane, $8 \mu \mathrm{m}$ wide beams). Device $\mathrm{C}$ has a larger membrane $\left(100 \times 100 \mu \mathrm{m}^{2}\right.$ membrane $)$ and actuator area, and therefore higher membrane displacements for the same $V_{\text {act. }}$ (b) FEM simulation of the fundamental resonance mode of a MEMS z-scanner of type A $(218 \mathrm{kHz})$.

damental resonance at $189 \mathrm{kHz}$ (Fig. 3), not far from the calculated $218 \mathrm{kHz}$. The simulations have also been used to predict the characteristics of a new series of MEMS z-scanner designs and to optimize them with respect to resonance frequency and actuation response. According to our FEM calculations, this new generation of MEMS z-scanners should have resonance frequencies up to $1.5 \mathrm{MHz}$.

The current generation of MEMS scanners cannot be used well in liquids because the liquid can cause a multitude of detrimental effects when it is present between the mem-

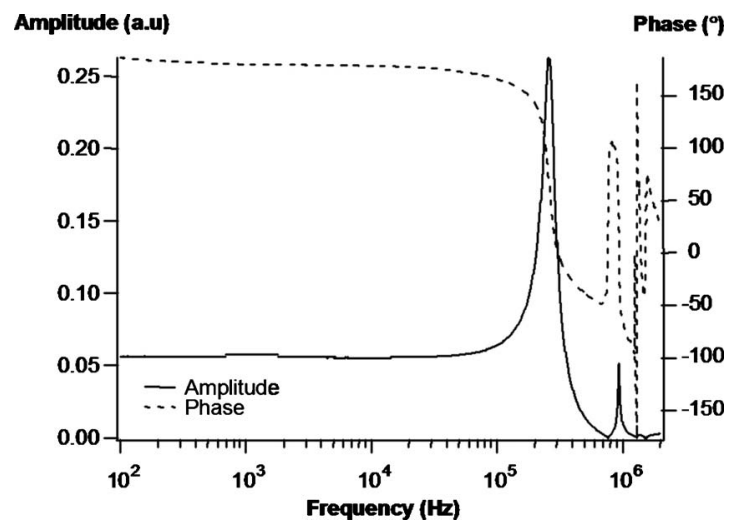

FIG. 3. Optical deflection measurement of a MEMS scanner response curve (Ref. 14). 

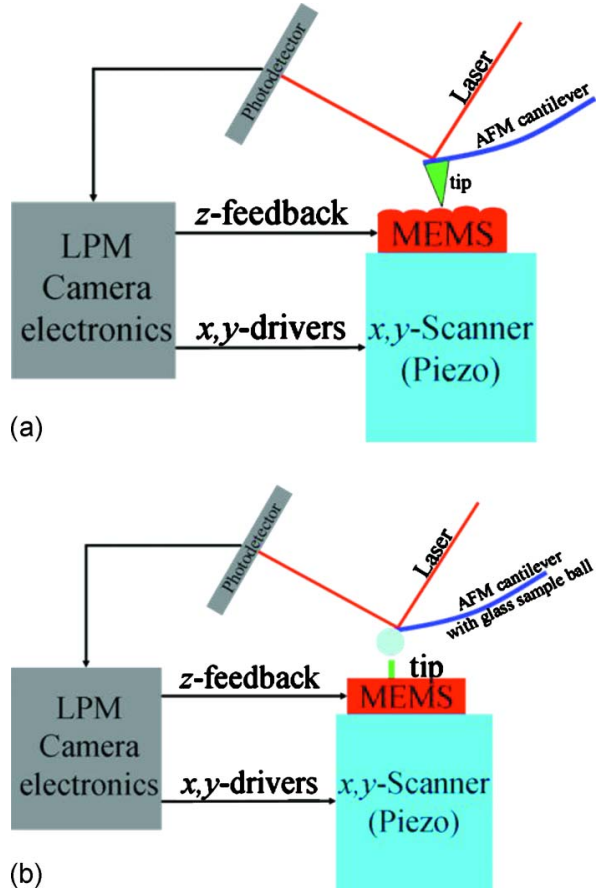

FIG. 4. (Color online) (a) The configuration of MEMS AFM experiment 1. (b) The configuration of MEMS AFM experiment 2.

brane and the actuation plate. If the liquid is water, and hence conductive, it may short the actuation voltage. The liquids' relative permittivity of more than one will change the voltage/displacement curve, and the increased squeeze film damping will also alter the response significantly. For use in liquid, we envision a MEMS scanner which either has an impermeable isolated moving membrane in contact with the liquid, or a setup where only the (longer) tip sticks into the liquid.

\section{MEMS SPM EXPERIMENTS}

The performance of the MEMS z-scanner has been investigated in two illustrative AFM experiments and a preliminary STM test. The AFM experiments are different with respect to the sample position [the sample is located either on the MEMS membrane or on a commercial AFM cantilever, Figs. 4(a) and 4(b)] but similar in other respects. The AFM experiments were performed with a Digital Instruments MultiMode AFM where the commonly used piezotube performs the $\mathrm{x}, \mathrm{y}$-scanning motion and Leiden Probe Microscopy (LPM) camera electronics drives both the piezoscan tube and the MEMS z-scanner. A conventional cantilever is used to measure the tip sample forces.

We have characterized this innovative combined setup in terms of vertical speed, vertical acceleration performance, height-to-deflection ratio (a measure for the feedback effectiveness), and maximum horizontal tip speed during scanning.

\section{A. MEMS scanner AFM experiments \\ 1. The MEMS as both sample and z-stage (experiment 1)}

Figure 4(a) shows a schematic drawing of the first experiment in which the polysilicon MEMS membrane surface

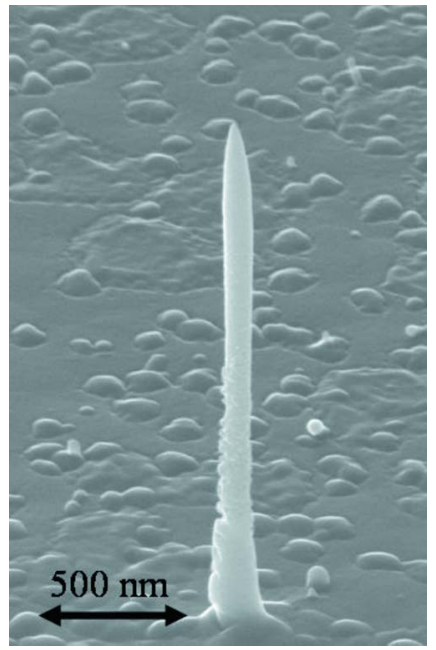

FIG. 5. (Color online) SEM micrograph of a $3 \mu \mathrm{m}$ long EBID platinum tip on a MEMS scanner.

itself is used as a sample and z-scanner. In this case the sample choice is limited to objects that can be deposited or placed on the MEMS membrane without destroying the device. Samples are, therefore, severely limited in both size and mass, but the full potential of the scanner can be exploited. With a peak-to-peak roughness of about $30 \mathrm{~nm}$ on a scan area of $1 \mu \mathrm{m}^{2}$, the polysilicon surface of the scanner itself is very suitable to test the feedback performance of the MEMS z-scanner.

\section{The MEMS scanner with probing tip (experiment 2)}

The range of sample choice is greatly enlarged by growing a tip on the MEMS membrane surface and mounting the sample on the AFM cantilever [Fig. 4(b)]. An in-house developed electron beam induced deposition (EBID) process using $\mathrm{Pt}\left(\mathrm{PF}_{3}\right)_{4}$ as a precursor gas ${ }^{15,16}$ was used to deposit a platinum tip in the middle of the membrane (Fig. 5). Tips deposited in this way can grow up to $6 \mu \mathrm{m}$ and have a tip radius of curvature of $\sim 20 \mathrm{~nm}$. The tip on the z-scanner used for the MEMS AFM experiments presented here is $3 \mu \mathrm{m}$ long.

The sample, a glass ball with a diameter of $\sim 100 \mu \mathrm{m}$, was mounted on the AFM cantilever by first dipping the cantilever in epoxy glue and subsequently pushing it onto the glass ball.

\section{B. MEMS AFM results}

In Figs. 6-8, AFM scans are given of the surface of the MEMS membrane, made during experiment 1, and in Fig. 9 an AFM scan is given of the surface of the glass ball, obtained during experiment 2. The details of the scans are given in Table I.

\section{Membrane velocity and acceleration performance (experiment 1)}

The polysilicon grains that constitute the surface of the membrane demand a high-speed feedback reaction. Vertical accelerations of up to $90 \mathrm{~m} / \mathrm{s}^{2}$ have been found at the vertical motion turning points between grains with the vertical 


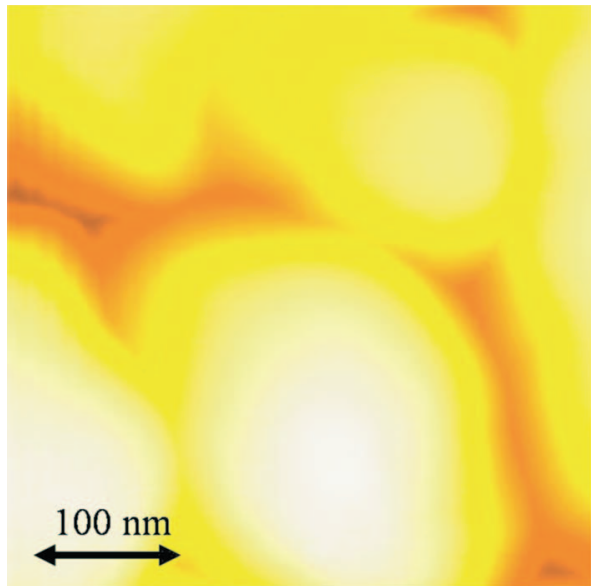

FIG. 6. (Color) Height and deflection image of the polysilicon MEMS membrane surface, experiment 1 .

velocity of the membrane at some points during the scan exceeding $1 \mathrm{~mm} / \mathrm{s}$. The vertical displacements of the membrane were calibrated with the help of the membrane displacement curve presented in Fig. 2(a).

\section{In-plane scan speed and feedback performance (experiment 1)}

The ratio of the peak-to-peak height and the peak-topeak deflection is a good measure for the feedback performance of the SPM system as a whole. In Fig. 6 we present a $0.4 \times 0.4 \mu \mathrm{m}^{2}$ scan of the MEMS membrane surface obtained from experiment 1 . The peak-to-peak height in this scan is 300 times larger than the peak-to-peak deflection measured, which indicates that the feedback with the MEMS scanner is performed properly and that the motion of the MEMS membrane follows the surface topology very well.

The image series in Fig. 7 shows how feedback performance changes with increasing tip speed. At a high horizontal tip speed [Fig. 7(d)], the image becomes highly deformed by in-plane resonances of the piezoscanner. Thus, the line

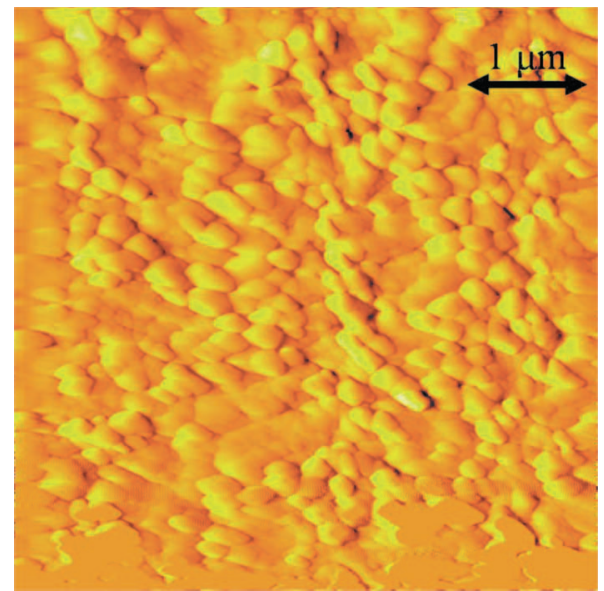

FIG. 8. (Color) Height image of the MEMS membrane surface, experiment 1 , at $1.05 \mathrm{~s}$ per frame. Small high frequency MEMS scanner resonances are visible in the image.

speed is not limited in this experiment by the fast moving MEMS z-scanner, which can follow all the details of the surface profile with ease. Instead, it is limited by the performance of the piezo, even though it has just to provide the relatively slow in-plane scanning motion.

Only when the MEMS device hits the voltage limit of its driver electronics, small resonances around $210 \mathrm{kHz}$ became apparent (Fig. 8) while scanning at a horizontal tip speed of $5 \mathrm{~mm} / \mathrm{s}$ [compare the resonance frequency with that of Fig. 7(d) of the piezo, which has a comparable in-plane tip speed].

\section{MEMS with integrated EBID tip (experiment 2)}

In Fig. 9 an image is shown of the surface of the glass ball, acquired in experiment 2 . In this case the peak-to-peak height is ten times larger than the peak-to-peak deflection. At a tip speed of $2 \mathrm{~mm} / \mathrm{s}$, a large resonance of $32 \mathrm{kHz}$ was observed in the images. FEM simulations have shown that this frequency matches the resonance frequency of the can-
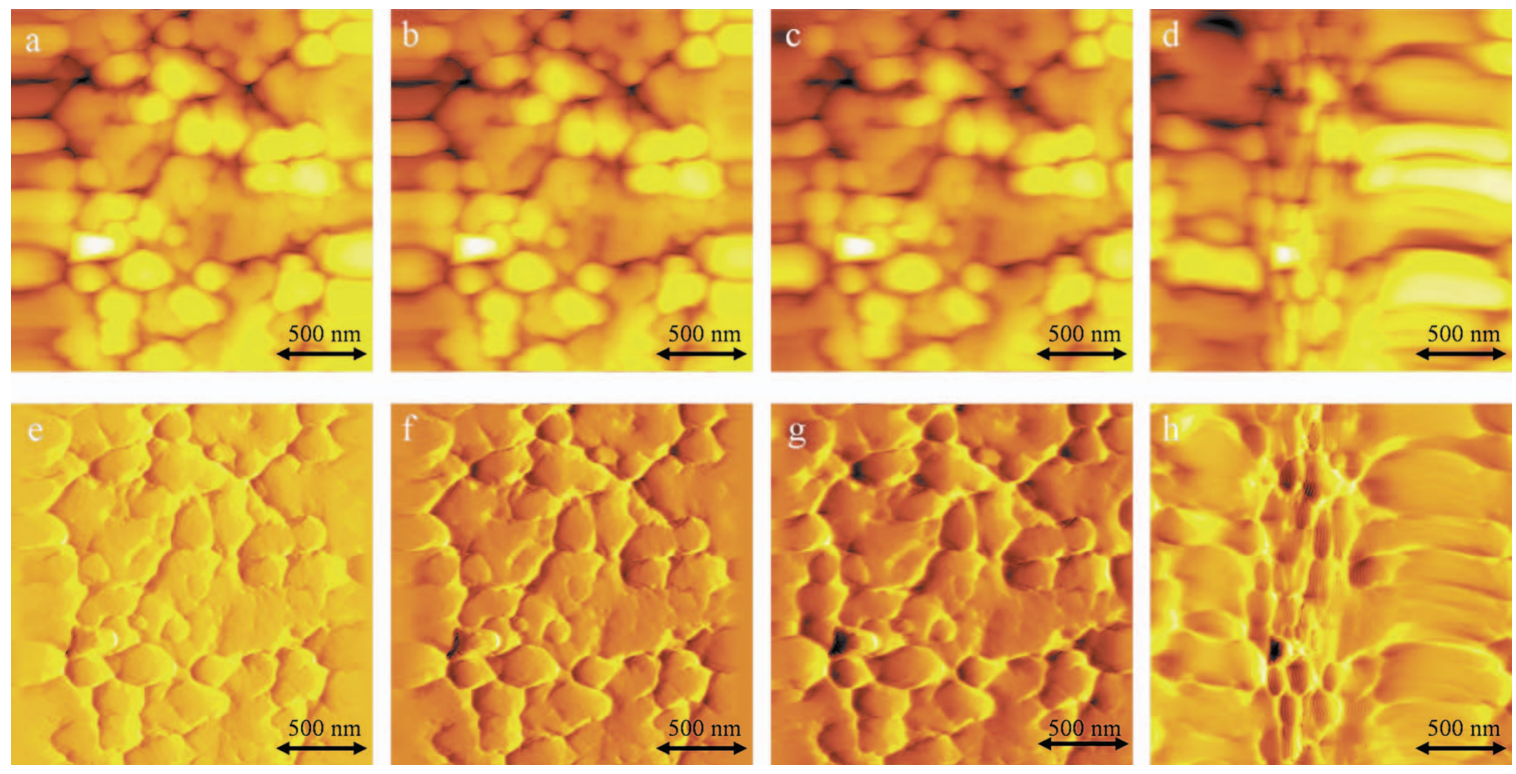

FIG. 7. (Color) Height images [above (a)-(d)] and deflection images [below (e)-(h)] of the MEMS membrane surface, experiment 1. 


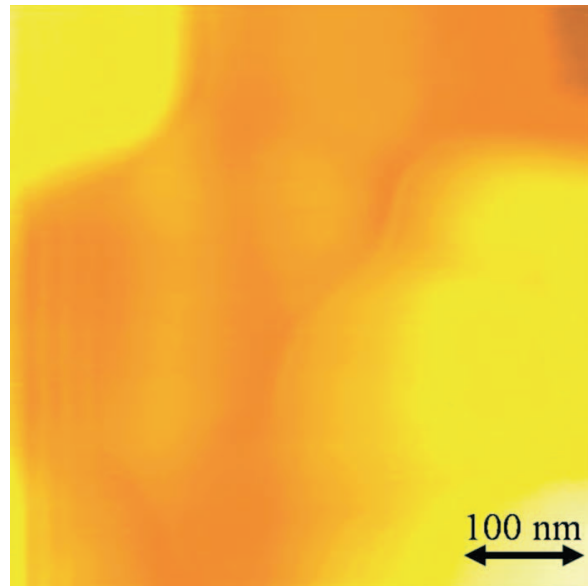

FIG. 9. (Color) Height image of the surface of the glass ball, experiment 2.

tilever with the glass ball glued to it. Because of this resonance, we could not scan as fast as in experiment 1. Still, these results show the functionality of the MEMS as AFM z-scanner with tip, as it is not the scanner but the AFM cantilever that resonates in this case.

\section{MEMS scanner STM experiments}

For testing the suitability of the MEMS scanner in a STM setup, a MEMS scanner with a deposited EBID platinum tip was also incorporated in a Nanosurf EasyScan STM. A small graphite flake was placed on a regular EasyScan tip to serve as the sample. The die with the MEMS z-scanner was placed on the EasyScan sample holder, and the regular coarse approach of the system was used to bring the graphite sample and the EBID tip on the MEMS scanner together. The tunneling current from the graphite sample to the EBID tip was recorded to verify the suitability of the scanner/tip combination for STM.

Contrary to the AFM experiments, where we completely controlled the scanning with our own electronics, in the EasyScan system it is not possible to intervene in the feedback system. Therefore, it was not possible to perform the actual feedback motion with the MEMS z-scanner. Instead, we had the EasyScan performing feedback with its own scanner, and, simultaneously, actuated the MEMS membrane with a square wave signal. This way, we could observe the MEMS membrane switching up and down, which is the same type of motion it would perform if used as a z-scanner. Figure 10 shows the image obtained in which the periodic switching of

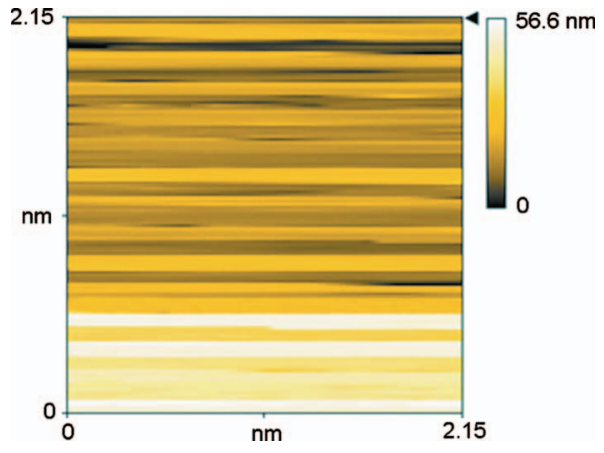

FIG. 10. (Color) The actuation of the MEMS z-scanner membrane in the Nanosurf EasyScan experiment shows that the MEMS scanner with EBID tip is suitable for STM as well as AFM.

the MEMS z-scanner is clearly visible. This proves two important facts about the MEMS scanner. First, the native silicon oxide, which is present between the polysilicon surface and the EBID tip, does not introduce problems when measuring the tunneling current. Second, the conductivity of the EBID platinum tip is high enough to perform tunneling experiments.

\section{DISCUSSION}

\section{A. High-speed SPM}

With the MEMS AFM experiments we have shown that the current generation of MEMS z-scanners is able to follow the surface accurately with tip speeds up to $5 \mathrm{~mm} / \mathrm{s}$ on the rough MEMS membrane surface. If the line speed limitation, which is now still set by the piezo-x,y-scanner, would be optimized, this MEMS-based setup can already produce the fastest image rates with full feedback currently available. For a smaller scan size in the nanometer range, the MEMS scanner could be used to acquire thousands of images per second at a tip speed of $5 \mathrm{~mm} / \mathrm{s}$ if piezos can cope with the lateral scan requirements.

We are currently working on a second generation of scanners. Finite element simulations have shown that adjustments to the design of the MEMS z-scanner could increase its resonance frequency even further to above $1.5 \mathrm{MHz}$, while maintaining a scan range of several hundreds of nanometers in the z-direction. This enables even higher tip speeds and frame rates up to tens of frames/s with a large scan size and range such as in Fig. 8.

TABLE I. Scan settings of the different figures.

\begin{tabular}{lccccc}
\hline \hline & $\begin{array}{c}\text { Scan size } \\
(\mu \mathrm{m})\end{array}$ & Tip speed & $\begin{array}{c}\text { Frame time } \\
(\mathrm{s})\end{array}$ & $\begin{array}{c}\text { Height }(\mathrm{p}-\mathrm{p}) \\
(\mathrm{nm})\end{array}$ & $\begin{array}{c}\text { Deflection }(\mathrm{p}-\mathrm{p}) \\
(\mathrm{nm})\end{array}$ \\
\hline Figure 6 & $0.4 \times 0.4$ & $40 \mu \mathrm{m} / \mathrm{s}$ & 10.5 & $\sim 85$ & 0.28 \\
Figure 7(a) & $2 \times 2$ & $0.4 \mathrm{~mm} / \mathrm{s}$ & 5.1 & $\sim 97$ & 7.2 \\
Figure 7(b) & $2 \times 2$ & $0.8 \mathrm{~mm} / \mathrm{s}$ & 2.6 & $\sim 96$ & 9.0 \\
Figure 7(c) & $2 \times 2$ & $2 \mathrm{~mm} / \mathrm{s}$ & 1.0 & $\sim 89$ & 16 \\
Figure 7(d) & $2 \times 2$ & $4 \mathrm{~mm} / \mathrm{s}$ & 0.5 & $\sim 79$ & 28 \\
Figure 8 & $5 \times 5$ & $5 \mathrm{~mm} / \mathrm{s}$ & 1.05 & $\sim 133$ & 25 \\
Figure 9 & $0.5 \times 0.5$ & $0.3 \mathrm{~mm} / \mathrm{s}$ & 1.6 & 18 & 1.8 \\
\hline \hline
\end{tabular}




\section{B. Tip considerations}

The EBID deposition technique produces a tip that is suitable for both AFM and STM imaging. We have shown that the conductivity of the tip is high enough for STM. For optimizing the tips further we are working in two directions. The EBID tips that were produced have a radius of around $20 \mathrm{~nm}$, which can be improved for high-resolution imaging by etching the tip or focused ion beam milling. Another important aspect of the EBID tip is that it is the only part of the production of the MEMS scanner that is not performed in a batch: every single tip has to be individually grown, while the rest of the MEMS scanner production process happens on the wafer level. Therefore, our future research will also be focusing on a suitable batch process for tip growth.

If the tip is eccentrically positioned on the membrane, which deforms upon actuation, as shown in Fig. 2(b), this may result in image deformation. This effect is not large though: in our experiment, the tip was placed within $6 \mu \mathrm{m}$ from the center of the membrane. At this distance to the center, the deformation of a typical membrane is so small $(<0.4 \%$ of the membrane displacement) that the horizontal disposition of the tip is 200 times smaller than the displacement of the membrane in the vertical direction. Therefore a swaying tip caused by membrane deformation will not lead to noticeable image deformation. In critical applications, the tip positioning can of course be much more accurate if required.

\section{CONCLUSION}

We have developed a new MEMS z-scanner that is suitable for high-speed SPM systems. The high velocities and accelerations the membrane is capable of show that our firstgeneration MEMS z-scanner can already control the tipsample distance perfectly at scan speeds comparable to the fastest state-of-the-art piezostack scanners.

The good feedback performance of the whole SPM setup including the MEMS scanner has been confirmed by the good ratios between peak-to-peak height and peak-to-peak deflection obtained from AFM experiments. Using the MEMS scanner, maximum tip speeds of $5 \mathrm{~mm} / \mathrm{s}$ can be reached without inducing resonances in the MEMS $\mathrm{Z}$-scanner, nor are resonance frequencies of the mechanical loop excited due to the extremely low moving mass. Cur- rently, the performance of our MEMS AFM system is limited only by resonances in the piezoscanner which performs the relatively slow $\mathrm{x}, \mathrm{y}$-scanning.

We have incorporated EBID deposited scan tips on the MEMS device and have shown that such an assembly is not only suitable for AFM experiments, but that the conductivity is high enough for STM operation as well. Further research will focus on the optimization of the MEMS z-scanners with respect to resonance frequencies and scan range and the incorporation of the MEMS z-scanner on a fast piezo-x,y-scan stage as well as on tip fabrication optimization.

${ }^{1}$ H. Yamashita, N. Kodera, A. Miyagi, T. Uchihashi, D. Yamamoto, and T. Ando, Rev. Sci. Instrum. 78, 083702 (2007).

${ }^{2}$ T. Fukuma, Y. Okazaki, N. Kodera, T. Uchihashi, and T. Ando, Appl. Phys. Lett. 92, 243119 (2008).

${ }^{3}$ G. Schitter, P. J. Thurner, and P. K. Hansma, Mechatronics 18, 282 (2008).

${ }^{4}$ L. M. Picco, L. Bozec, A. Ulcinas, D. J. Engledew, M. Antognozzi, M. A. Horton, and M. J. Miles, Nanotechnology 18, 044030 (2007).

${ }^{5}$ M. J. Rost, L. Crama, P. Schakel, E. van Tol, G. B. E. M. van VelzenWilliams, C. F. Overgauw, H. ter Horst, H. Dekker, B. Okhuijsen, M. Seynen, A. Vijftigschild, P. Han, A. J. Katan, K. Schoots, R. Schumm, W. van Loo, T. H. Oosterkamp, and J. W. M. Frenken, Rev. Sci. Instrum. 76, 053710 (2005)

${ }^{6}$ P. Vettiger, G. Cross, M. Despont, U. Drechsler, U. Durig, B. Gotsmann, W. Haberle, M. A. Lantz, H. E. Rothuizen, R. Stutz, and G. K. Binnig, IEEE Trans. Nanotechnol. 1, 39 (2002).

${ }^{7}$ P. F. Indermuehle, C. Linder, J. Brugger, V. P. Jaecklin, and N. F. de Rooij, Sens. Actuators, A 43, 346 (1994).

${ }^{8}$ P. F. Indermühle, V. P. Jaecklin, J. Brugger, C. Linder, N. F. de Rooij, and M. Binggeli, Sens. Actuators, A 47, 562 (1995).

${ }^{9}$ Y. Xu, N. C. MacDonald, and S. A. Miller, Appl. Phys. Lett. 67, 2305 (1995).

${ }^{10}$ J. Carter, A. Cowen, B. Hardy, R. Mahadevan, M. Stonefield, and S. Wilcenski, PolyMUMPs Design Handbook (MEMSCAP, 2008); see www.memscap.com.

${ }^{11}$ F. C. Tabak, E. C. M. Disseldorp, T. H. Oosterkamp, A. J. Katan, M. B. S. Hesselberth, J. W. M. Frenken, and W. M. van Spengen, Symposium on Surface Science, St. Moritz, Switzerland, 2009.

${ }^{12}$ G. M. Rebeiz, RF MEMS Theory, Design and Technology (WileyInterscience, Hoboken, 2003).

${ }^{13}$ W. M. van Spengen, R. Puers, R. Mertens, and I. De Wolf, J. Micromech. Microeng. 14, 514 (2004).

${ }^{14}$ M. J. Rost, G. J. C. van Baarle, A. J. Katan, W. M. van Spengen, R. Schakel, W. A. van Loo, T. H. Oosterkamp, and J. W. M. Frenken, Asian J. Control 11, 110 (2009).

${ }^{15}$ A. Botman, M. B. S. Hesselberth, and J. J. L. Mulders, Microelectron. Eng. 85, 1139 (2008).

${ }^{16}$ A. Botman, M. B. S. Hesselberth, and J. J. L. Mulders, J. Vac. Sci. Technol. B 26, 2464 (2008). 